\title{
Research on Internet of Things Elevator Fault Detection and Automatic Alarm System Based on Android
}

\author{
Juanjuan Li \\ Shaanxi Institute of International Trade \& Commerce, Xi’an, Shaanxi, 712046
}

\author{
Keywords: IOT, Elevator Fault, Automatic Alarm System
}

\begin{abstract}
In recent years, the domestic elevator installation continues to expand and the increasing number of aging elevators led to frequent elevator failures, and now a large number of aging elevators did not reach the design life is still in the front line, and this part of the elevator generally lack of elevator fault monitoring and sleepy Alarm equipment, once the maintenance is not in place, the elevator is prone to failure to stop the ladder and the problem of sleepy people, when the fault occurs, the elevator and the elevator personnel inside information can not be effectively passed to the maintenance and rescue workers, leading to the elevator fault repair and sleepy events The treatment is not timely enough, easily lead to emotional distress trapped staff, leading to tragedies. In order to ensure the safety of people's lives and property, it is of practical significance to design a universal fault monitoring and alarm system for all aged elevators of different brands.
\end{abstract}

\section{Introduction}

The status of elevator operation is directly related to the life and property safety of the general public. Therefore, elevator operation safety has been paid attention to by all walks of life. Nowadays, the early-installed elevators gradually enter the middle and later stages of the lifespan of the elevator design, which is already an aging elevator. With the wear and tear of the parts and components, the operational reliability is gradually declining, the mean time between failures is greatly shortened and some of the masses have started to be affected with the passage of time, the number of elevators entering the aging phase is also constantly expanding, which directly causes the frequent elevator accidents in recent years and seriously endangers the lives and property of the people. According to published statistics, only 3,066 elevator accidents occurred in Hangzhou City in 2011, causing a lot of casualties and economic losses. With the continuous increase in the number of aging elevators, elevator safety operation, fault monitoring, fault alarm and elevator maintenance have also been elevator manufacturers, properties and people's attention.

\section{Elevator fault monitoring and sleepy alarm system design}

The normal operation of the elevator requires hundreds of well-functioning machines. The electrical components cooperate with each other to ensure that the monitoring system needs to monitor more than ten states. Among them, the parameters that directly affect the safe operation of the elevator include the running status of the car, the status of the car, the status of the car door, whether the main loop of the car is working, whether there are people in the car, whether someone triggers the alarm button or not. According to the technical stipulations in the contract, the elevator fault detection and the installation of alarming system equipment shall not change the original mechanical structure of the elevator and shall not intervene in the control loop of the elevator, and shall not affect the normal running status of the elevator. Therefore, for this system, the optimal solution is to use a set of sensors independent of the elevator electromechanical system to monitor the necessary status information during the operation of the elevator. Here you can initially determine the composition of the system structure.

The detection of these conditions are all independent of the elevator electromechanical system sensors, the sensor is connected to the signal through the cable mounted on the top of an embedded control terminal, the control terminal through the sensor on the lift the above state parameters 
collected. Monitoring terminal built-in wireless communication module, the detection data can be judged and processed, and then sent to the residential property monitoring computer, monitoring computer to monitor the terminal data uploaded by multiple unified management, including elevator operating status information, maintenance information, annual inspection information, If the elevator fails, it will automatically trigger the emergency response mechanism to classify the type of failure. If passengers are trapped, it is regarded as the most urgent event. The first time, the information about the trapped persons and the current status of the elevator will be provided to support the rescue operation. If the general failure, then start the general handling mechanism, coordinating staff placed warning signs to inform the public lift failure, and timely maintenance personnel maintenance and repair. A monitoring computer in a residential area and multiple monitoring terminals in a community form a regional monitoring system. A larger monitoring and measuring system is formed by each system module and is uniformly used and managed by the regulatory authority. The system can realize more Features.

\section{Elevator fault monitoring and sleepy alarm system hardware design}

In order to ensure the reliability and stability of the hardware system, the following principles should be noted in the system hardware design:

The performance of the master chip is directly related to the performance of the monitoring terminal. The reliability of the chip can improve the reliability of the monitoring terminal so as to improve the reliability of the monitoring system. The comprehensive chip interface greatly simplifies the design of the circuit and the design difficulty, as well as reducing the programming workload. For example, the main chip with the hardware IIC can significantly reduce the programming workload of the chip without the hardware IIC.

Today, the pace of technological progress is faster, user needs are constantly increasing, sensor updates, and changes in the number and types of sensors should be taken into account in hardware design, with changes to the program structure and modification of the algorithm for subsequent software programming of the space, so that the system hardware and software design more flexible, in order to adapt to different elevators in different environments, provides the possibility. Modular development, can facilitate the introduction of other development forces, software programming can be used collaborative design model to improve efficiency.

Circuit board PCB layout will directly affect the reliability of their own and other systems and anti-interference. Good trace design can improve the reliability and anti-interference, poor design not only self-interference, but also generate unnecessary electromagnetic radiation, interference with the work of other nearby environmental circuitry. To ensure its own circuit performance, PCB printed circuit board design should be reasonable use of copper wiring, to ensure that the circuit has good EMC (Electromagnetic Compatibility) to minimize EMI (electromagnetic interference) index.

Because the entire monitoring and alarm terminal requires the ability to operate in power-off mode, the battery is internally designed to ensure that the monitoring terminal can operate reliably for a certain period of time after the power is cut off. Therefore, reducing overall system power consumption is the best way to improve battery life and extend battery life. Therefore, in the chip selection, the power consumption of the chip should be fully considered and the efficiency of the use of power, circuit design, as much as possible for some modules design power control interface, in order to achieve idle dormancy to provide the principle of standby.

Has been put into operation in the elevator, regardless of the construction space, and the ladder to ensure equipment construction time is very limited, so the monitoring terminal should be compact compact size, external sensor wiring design should take into account the simple and quick, reliable and effective principle.

\section{Monitoring Terminal Software Design}

C8051F020 support JTAG hardware online debugging, in the process of simulation and debugging, can call the chip resource monitoring and debugging module, such as: cross-switch 
monitoring configuration module, serial port monitoring configuration module, timer monitoring configuration module, AD / DA converter monitoring configuration module, Etc. Users can dynamically configure the value of hardware registers or ports through these modules. Users can even view the contents of $C$ language variables during program running, which greatly improves the convenience of debugging and improves the efficiency of program development. However, in order to achieve these functions, you need to use Silicon Lab's U-EC5 emulator, in addition to installing the appropriate USB driver, you need to install the debugger's KEIL support package, the support package must be installed to KEIL software installation directory Under the installation, but also in the Project menu bar select Options for Target to enter the target configuration dialog box, in the USE drop-down list box, select Silicon Labs C8051Fxxx Driver support.

Because the monitoring terminal is composed of many modules, the corresponding communication and event handling functions are more. In order to facilitate the modification and search of the code writing process, different function modules are categorized and stored in different $\mathrm{C}$ code documents. Project files in accordance with the relevant hardware module divided Init.c, Mod Bus.c, RFID.c, MC39i.c, MPU6050.c, RTC.c, Main.c a total of seven C code documents, each document is stored Hardware module to deal with the basic functions of these basic function modules in accordance with the design flow of the final composition of the monitoring terminal control procedures. The file is described as follows: The Init.c document mainly stores the set of processing functions related to hardware initialization. After the hardware is powered on, the hardware modules including the control core do not have the conditions for executing the main code. Therefore, before executing the main code, the hardware modules are configured to match their status with the expected design features, followed by the main code to complete the system design functions. The Mod Bus.c document mainly stores the processing functions related to the communication interface. In this system, each of the hardware modules adopts more communication interface standards, such as a universal serial interface, an IIC interface, an SPI interface, a single bus interface, etc. The MCU mainly uses these Interface functions and other functional modules to achieve data communication, data acquisition and command control. RFID.c document mainly stores the radio frequency card reader control related processing functions, the radio frequency card reader also has a micro-controller inside, the microcontroller chip has a code written in the code, there is a function code in the table, the corresponding table Within each instruction has a simple processing function, C8051F020 issued by the SPI bus a sequence of instructions, the microcontroller can be based on the sequence of commands to the combination of processing functions to complete a designated function, different instruction sequences correspond to different functions Therefore, most of the functions in the RFID.c file are mainly the functions of different functional instructions of the RF card reader, corresponding to functions such as card seeking, ID identification, password matching and IC card data reading and writing. MC39i.c file stored phone module control-related processing functions, MC39i also has a processor within the same, integrated AT instruction set, C8051F020 is also sent through the serial port standard AT control instructions, step by step to complete the module configuration, call, send text messages , Receive calls, receive short messages and other functions, therefore, most of the functions in this document is through the AT command operation MC39i module combination of functions, in addition to legitimate users to judge, SMS content analysis and judgment functions. The MPU6050.c file mainly stores the relevant processing functions of the acceleration sensor. The acceleration module, as a single sensor, has a single data function and a simple processing function. The functions in the file mainly fulfill the functions of acceleration sensor configuration, data reading, and judgment processing. The RTC.c file mainly stores the related processing functions of the real-time clock calendar module. The functions in the file mainly finish the time data extraction and arrangement, module configuration and time information modification of the real-time clock module.

\section{Conclusion}

The system design combines the two main Internet of Things technology: GPRS wireless remote 
transmission and RFID technology. The monitoring terminal uses an independent set of sensors to collect the running status of the elevator, identify the type of the fault according to the collected data and the summary judgment criteria, and add the RFID technology in the design to collect the information of the maintenance personnel and the inspectors. The GSM / GPRS module Use SMS or GPRS to connect the Internet to transmit the status parameters of the elevator. Voice communication enables full-duplex intercom between trapped persons and rescuers. The standby power and corresponding power management functions are added to the monitoring terminal to ensure the stable operation of the system over a long period of time, Real-time monitoring of elevator operation status.

\section{Acknowledgement}

Science and technology research project of Xianyang City,2017 NO.: 2017k02-10

Research on monitoring system of elevator internet of things based on Xianyang City

\section{References}

[1] Liu Songguo, Han Shuxin. Research on elevator operation status monitoring and fault remote alarm system [J]. Automation and Instrumentation. 2011 (10)

[2] Zhang Juli. PLC in the elevator control application [J]. Science \&Technology Information. 2009 (36)

[3] Tao Peng, Sun Xiaoming, Zhang Chao. An elevator fault diagnosis method based on neural network reasoning strategy [J]. Journal of Wuhan University of Technology (Information and Management Engineering), 2009 (06)

[4] Orgasm, Liu Anhou, Guo Yongcai. Remote elevator remote monitoring system based on multiple transmission technologies [J]. Computer Systems Applications. 2009 (11)

[5] Zhongzhao Feng. Remote control platform of city elevator based on internet environment [J]. Automation Technology and Application. 2009 (08)

[6] Li Yi, Zhang Huihui, Zheng Gang. Design and realization of intelligent elevator monitoring system [J]. Major of Manufacturing Automation. 2009 (08) 